\title{
Double éclairage sur l'organisation thématique de discours oraux publics
}

\author{
Grobet, Anne* \& Montemayor-Borsinger, Ann** \\ *Université de Genève, ELCF \\ anne.grobet@unige.ch \\ **Universidades Nacionales de Río Negro y de Cuyo, Argentine \\ aborsinger@unrn.edu.ar
}

\section{Introduction}

Notre analyse porte sur des discours oraux présentant différents degrés de formalité, impliquant un public séparé du locuteur spatialement (entretien télévisé) ou institutionnellement (cours ex cathedra). Il s'agit d'étudier l'organisation thématique de ces discours et de mettre en évidence leur spécificité, à la lumière de deux approches théoriques distinctes. Premièrement, la Linguistique Systémique Fonctionnelle (LSF) (Halliday 1994, Berry 1996, Halliday et Mathiessen 2004), qui considère le thème du point de vue positionnel comme le point de départ d'un énoncé («message »), autorise une analyse discursive globale et dynamique. La succession de ces points de départ constitue l'échafaudage du texte et indique comment l'auteur a choisi de le développer. Deuxièmement, nous présentons une analyse à l'aide d'une approche considérant le thème plutôt sous un angle informationnel (cf. notamment Chafe 1994, Lambrecht 1994), et impliquant une approche multidimensionnelle, voire multimodale, de l'organisation thématique, combinant la prise en compte d'informations lexicales, syntaxiques et prosodiques, avec des éléments relevant de la structure discursive (Roulet, Filliettaz \& Grobet 2001).

Notre corpus «pilote», qui figure en annexe, est constitué d'extraits de deux discours manifestant différents degrés d'oralité (Gadet 1996) autorisant une analyse contrastive : fragment d'un entretien télévisé entre Bernard Pivot et Georges Simenon, dans le cadre d'une émission d'Apostrophes, un discours public clairement ancré dans l'oral, suivi d'un extrait d'une leçon de Michel Foucault au Collège de France, dont le statut est plus ambigu : il y a d'une part le son, que nous avons transcrit et utilisé ici pour nos analyses, qui pourrait être qualifié « d'écrit oralisé », dans la mesure où il s'agit d'un discours planifié, reposant manifestement sur un texte écrit, sinon presque entièrement lu. D'autre part, il existe une transcription de ce même discours qui a fait l'objet d'une publication, avec quelques légers remaniements (l'écrit oralisé est «scripturalisé»). Nous laisserons de côté pour le moment la comparaison entre notre transcription et la version écrite, et baserons nos analyses sur la première pour permettre une comparaison cohérente avec le discours oral de Simenon.

D'un point de vue théorique, la notion de «thème» fait notoirement l'objet de multiples débats terminologiques et conceptuels (p. ex. Galmiche 1992, Mondada 1994). La thèse De l'ordre des mots dans les langues anciennes comparées aux langues modernes de Weil constitue un antécédent important dans l'étude de l'organisation thématique:

Il y a donc un point de départ, une notion initiale, qui est également présente et à celui
qui parle et à celui qui écoute, qui forme comme le lieu où les deux intelligences se
rencontrent; et une autre partie du discours, qui forme l'énonciation proprement dite.
Cette division se retrouve dans presque tout ce que nous disons.
(Weil $1844: 24$ )

Cette double définition du thème comme point de départ et notion initiale a été reprise par Mathesius, puis raffinée et séparée dans les années 60 du siècle dernier. Certains chercheurs tels que Firbas (1964, 1992) considèrent le thème du point de vue informationnel comme ce qui est connu, tandis que d'autres, comme le fait implicitement Daneš (1974) et comme le fait explicitement Halliday (1967), le considèrent du point de vue positionnel comme le point de départ du message qui introduit ce qui suit (Montemayor- 
Borsinger 2009). Dans ces deux visions du thème, ce qui ressort est le fait que généralement, pour des raisons fonctionnelles, le point de départ constitue l'information connue, suivie du rhème qui généralement constitue l'information nouvelle. Au cours des années, chacune de ces définitions a fait l'objet d'appropriations différentes, de diverses spécifications et/ou modifications. Il n'est pas inutile de noter, du côté des tenants de la perspective informationnelle, la réinterprétation cognitive de la dichotomie connu-nouveau, qui en vient à être considérée plutôt comme un continuum. Chafe (1994) distingue ainsi l'état d'activation, lié à la présence supposée par le locuteur d'une information dans la " conscience » de l'interlocuteur (une information pouvant être déjà active, semi-active ou inactive), de l'identifiabilité, concernant les hypothèse que le locuteur fait sur le statut des informations dans les connaissances générales de son interlocuteur (une information étant présentée comme identifiable ou non identifiable). Dans cette perspective, l'état d'activation et l'identifiabilité dépendent non seulement des informations présentes dans le cotexte, mais aussi de celles présentes dans le contexte.

C'est donc du point de vue de ce double éclairage positionnel et informationnel du thème, mobilisé par deux approches différentes, que nous nous proposons d'analyser l'organisation thématique d'extraits de discours oraux publics, dans une perspective contrastive.

\section{2 Éclairage positionnel de la Linguistique Systémique Fonctionnelle}

\subsection{Cadre théorique}

Pour la Linguistique Systémique Fonctionnelle (Halliday 1994, Halliday et Mathiessen 2004) la lexicogrammaire constitue un système de choix significatifs que fait le locuteur dans la construction de textes. Ces choix ne sont pas arbitraires mais servent à réaliser des fonctions sémantiques répondant à des contextes sociaux.

La LSF propose ainsi une vision tripartite multidimensionnelle de l'organisation du discours, justifiée par le fait que le langage réalise simultanément trois fonctions. La première concerne les représentations d'éléments dans le monde et les relations entre elles. Ces représentations sont encodées par des procès (ou verbes selon la terminologie grammaticale traditionnelle), des participants associés à ces procès, constituant la transitivité de la proposition, et éventuellement des circonstances associées (de temps, de lieu, de cause, etc.). Cette fonction de représentation est la " métafonction idéationnelle » - « méta » dans le sens d'une fonction qui va au-delà du lexique et de la grammaire d'une proposition isolée, et qui concerne la sémantique du discours pris dans sa totalité.

La seconde fonction concerne les relations sociales qui s'établissent au moment où le locuteur s'adresse à son interlocuteur et fait certains choix pour représenter le monde. Ces choix concernent par exemple le pronom personnel (vous ou $t u$ ), la conjugaison, le mode verbal et la modalité, qui constituent autant de traces lexicales et grammaticales liées à la « métafonction interpersonnelle ».

Finalement la "métafonction textuelle » concerne l'organisation des deux autres métafonctions pour former des chaînes d'éléments sémantiques qui constituent des textes. Ici la LSF fait une distinction entre la «structure thématique » et la «structure informationnelle ». Pour analyser la structure thématique, la LSF utilise le terme de "Thème », avec majuscule, pour marquer une perspective positionnelle rattachée à la notion de point de départ du message. La structure informationnelle quant à elle repose pour la LSF principalement sur de nombreux éléments qui relèvent du contexte extralinguistique.

Le Thème ici tient donc à l'ordre des mots et constitue un point de départ qui doit obligatoirement inclure un élément provenant de la métafonction idéationnelle, c'est-à-dire un participant ou une circonstance qui délimite le Thème à droite et à partir duquel se déroule le reste du message, le Rhème. Dans cette perspective positionnelle tout autre élément qui précède le premier participant ou circonstance, par exemple une conjonction, fait partie du Thème mais n'est pas un point de départ suffisamment constitué. Cette perspective est dynamique, dans la mesure où la succession des Thèmes fait progressivement émerger la sémantique du discours complet et permet de distinguer ce qui, en LSF, a été décrit comme la 
«méthode de développement» du discours (Fries 1983), son «échafaudage » (Stainton 1993), ou son « support » (Mendenhall 1990) pour les « apports » qui se font dans le Rhème.

En LSF, dans une proposition déclarative, si le Thème est fusionné avec le rôle de sujet, il s'agit d'un Thème non marqué. Si ce n'est pas le cas, il s'agit d'un Thème marqué, «marqué » entendu non pas dans le sens d'un choix inhabituel ou surprenant de la part de l'interlocuteur, mais dans celui d'un choix qui reflète la mise en avant de certaines motivations sémantiques fonctionnelles au développement du discours (Davies 1997). Le Thème marqué encadre et fait ressortir certaines séquences locales pour faciliter une meilleure interprétation du message par le destinataire, et signale des bifurcations dans le discours comme dans le texte de Foucault avec à ceci près. toutefois que ce n'est pas tout à fait du discours raciste / et dans le texte de Simenon avec c'est lui, où c'est est considéré comme un thème « sémantiquement vide » au niveau du discours, un argument pour inclure discours raciste et lui dans le Thème. En contrepartie le Thème non marqué maintient la continuité du topique du discours (voir le texte de Simenon qui est centré sur le je de l'émetteur du discours).

La LSF distingue aussi des Thèmes multiples. Nous venons de voir que la présence dans le Thème d'un élément idéationnel (participant ou circonstance) est obligatoire et délimite le Thème à droite. Cet élément idéationnel peut être précédé à gauche par des Thèmes de caractère textuel et/ou interpersonnel, qui sont facultatifs, comme les mais et euh qui précèdent je dans le texte de Simenon. Dans le cours de Foucault il y a plusieurs Thèmes interpersonnels de grande complexité. Ce sont des modalisateurs épistémiques tels que à ceci près. toutefois que ce n'est pas tout à fait dont l'auteur se sert pour qualifier la valeur de vérité du concept de discours raciste qui délimite le Thème à droite. Un exemple similaire provenant du même texte est je dirais plutôt qui exprime des réserves et qualifie l'élément idéationnel discours de la guerre ou de la lutte des races délimitant le Thème à droite.

Nous passerons maintenant à l'analyse thématique des différents choix faits par Simenon et Foucault dans la construction et la transmission de leurs discours respectifs.

\subsection{Analyses}

L'analyse du premier fragment nous montre comment Georges Simenon, en tant que personnalité énonciatrice dans un entretien télévisé, se choisit systématiquement comme point de départ. Le support de son discours est donc le pronom je, un point de départ « léger » et congruent avec la fonction de ce genre de discours éminemment personnel. 


\begin{tabular}{|l|l|l|}
\hline \multicolumn{2}{|l|}{ Support } & Apport \\
\hline Thème marqué & Thème non marqué & Rhème \\
\hline & je & suis un instinctif \\
\hline & je & ne suis pas du tout un intellectuel \\
\hline & euh je & n'ai jamais pensé un roman \\
\hline & j' & ai senti un roman \\
\hline & je & n'ai jamais pensé un personnage \\
\hline & j' & ai senti un personnage \ n'est-ce pas \\
\hline & euh je & n'ai jamais inventé une situation \\
\hline & la situation & est venue lorsque j'écrivais un roman \\
\hline & mais je & ne savais pas du tout où mon personnage allait me mener \\
\hline c'est lui & & qui me menait \\
\hline & et je & vivais ... dans la peau de ce personnage ... \\
\hline
\end{tabular}

Tableau 1. Analyse d'un extrait du discours de G. Simenon

L'échafaudage nécessairement beaucoup plus chargé du discours suivant, comparé avec le discours de G. Simenon, s'explique par sa grande formalité, probablement la plus extrême dans le genre de l'oral. Michel Foucault, au début de sa classe magistrale du 28 janvier 1976 au Collège de France, commence par s'adresser au vous de son auditoire, puis, malgré son choix explicite du je énonciateur, choix typique de l'oral, il prend ses distances. Ce je est moins fréquent que celui de G. Simenon, et différent : c'est un je généralement en position rhématique fortement accompagné de modalités épistémiques (marquées en gras dans le tableau) : j'ai voulu faire..., je crois qu'il faut..., je voulais faire..., j'aurais voulu vous montrer..., je voudrais vous parler... La nature de ce genre ex cathedra utilise la modalité épistémique pour une argumentation théorique qui cherche à tempérer un discours qui, dans le cas du fragment, se réfère au cours précédent. Cette modalité est d'ailleurs renforcée dans le support du discours par une succession de Thèmes marqués dont la fonction est de mettre en contexte et préciser les concepts de discours raciste, d'éloge, et de contre histoire:

\begin{tabular}{|l|l|l|}
\hline \multicolumn{2}{|l|}{ Support } & Apport \\
\hline Thème marqué & $\begin{array}{l}\text { Thème non } \\
\text { marqué }\end{array}$ & Rhème \\
\hline bon vous & $\begin{array}{l}\text { avez pu croire que j'ai entrepris/ la dernière fois/ de } \\
\text { vous faire : l'histoire et l'éloge du discours raciste } \backslash\end{array}$ \\
\hline $\begin{array}{l}\text { à ceci près . toutefois que ce } \\
\text { n'est pas tout à fait du discours } \\
\text { raciste / }\end{array}$ & vous & n'avez pas eu tout à fait tort/ \\
\hline $\begin{array}{l}\text { je dirais plutôt du discours de la } \\
\text { guerre ou de la lutte des races } \backslash\end{array}$ & dont j'ai voulu faire l' éloge et 1'histoire / \\
\hline
\end{tabular}




\begin{tabular}{|c|c|}
\hline & $\begin{array}{l}\text { épi un épisode particulier euh localisé de : ce grand } \\
\text { discours de la guerre de la lutte }: \text { des races } \backslash\end{array}$ \\
\hline le discours raciste & $\begin{array}{l}\text { c'en a été un épisode /une phase / c'en a été à vrai } \\
\text { dire plutôt même . le retournement / la reprise en tout } \\
\text { cas / à la fin du XIXe siècle / du discours de la guerre } \\
\text { des races / une reprise de ce vieux discours / dèjà } \\
\text { séculaire à ce moment là / dans des termes socio- } \\
\text { biologiques / et ceci / à des fins euh essentiellement } \\
\text { de conservatisme social / et dans un certain nombre } \\
\text { de cas au moins / de domination coloniale } \|\end{array}$ \\
\hline $\begin{array}{l}\text { ceci étant dit / pour situer un peu } \\
\text { là à la fois le lien et la différence }\end{array}$ & $\begin{array}{l}\text { / entre discours raciste et discours de la guerre des } \\
\text { races /eh bien c'est bien l'éloge / de ce discours de la } \\
\text { guerre des races que je voulais faire / }\end{array}$ \\
\hline l'éloge / & $\begin{array}{l}\text { en ce sens que j'aurais voulu vous montrer / euh } \\
\text { comment / pendant un temps / au moins / c'est à dire } \\
\text { jusqu'à la fin du XIXe siècle / jusqu'au moment où il } \\
\text { se retourne en un discours raciste / eh bien ce } \\
\text { discours de la guerre des races a fonctionné comme } \\
\text { une contre histoire } \backslash\end{array}$ \\
\hline $\begin{array}{l}\text { et c'est un petit peu de cette } \\
\text { fonction de contre histoire / }\end{array}$ & que je voudrais vous parler aujourd'hui. \\
\hline
\end{tabular}

Tableau 2. Analyse d'un extrait du discours de M. Foucault

Cette succession de Thèmes marqués centrés sur des concepts abstraits et accompagnés d'une modalisation épistémique reflète donc la préoccupation de M. Foucault de guider son auditoire afin qu'il comprenne son approche et puisse situer ce qu'il a dit antérieurement ainsi que ce qu'il va dire. Cet échafaudage fortement ancré dans la rhétorique universitaire est en contraste flagrant avec l'échafaudage du premier fragment. Tandis que G. Simenon maintient les points de départ sur son je d'énonciateur qui donne ses opinions sans les modaliser pour se montrer comme un instinctif et non pas comme un intellectuel, M. Foucault choisit des notions abstraites et emploie la modalité épistémique pour éviter des malentendus, convaincre l'auditoire et anticiper de possibles critiques.

\section{3 Éclairage informationnel d'une approche multidimensionnelle}

\subsection{Cadre théorique}

La prise en compte de la dimension informationnelle de la notion de thème implique un angle d'analyse nécessairement multidimensionnel. En effet, le caractère donné - ou "déjà actif », dans les termes de Chafe (1994) - d'une information peut être rattaché au contexte (p. ex. les interlocuteurs constituent par leur présence des informations constamment disponibles), au cotexte (évocation d'une information dans le discours antérieur), au marquage lexical, syntaxique, et prosodique, sans parler même des éventuels signaux mimo-gestuels que nous ne pourrons discuter ici. Pour en rendre compte, nous emprunterons des éléments théoriques à différents modèles, et notamment à l'approche modulaire et interactionniste de l'organisation du discours développée dans Roulet, Filliettaz \& Grobet (2001), sans toutefois nous astreindre à adopter une méthodologie strictement « modulaire ».

Dans cette approche, le «topique ${ }^{1}$ » est considéré comme un «point d'ancrage » permettant l'introduction d'information nouvelle, par chaque unité discursive minimale (appelée "acte»). Ce topique est considéré comme une information que le locuteur suppose être non seulement identifiable pour l'interlocuteur, mais aussi déjà active dans sa mémoire immédiate. Ceci dit, il existe pour chaque 
interaction un grand nombre de points d'ancrage, manifestant différents degrés de pertinence et de saillance pour les interlocuteurs. Brown \& Yule (1983) proposent de regrouper dans la notion de «cadre topical» (topical framework) l'ensemble des informations, introduites par le contexte ou le cotexte, susceptibles de fonctionner comme topique d'un segment discursif donné :

The topic framework consists of elements derivable from the physical context and from the discourse domain of any discourse fragment. Notice that we have concentrated on only those elements which are activated, that is, relevant to the interpretation of what is said. (Brown \& Yule 1983: 70)

Les auteurs soulignent qu'un tel cadre topical permet d'évaluer la pertinence topicale de ce qui est dit.

A partir du moment où l'ensemble des informations identifiables et actives ou semi-actives est pris en considération, on ne peut manquer d'observer le grand nombre des topiques possibles. Or, Lambrecht (1994) tout comme Roulet, Filliettaz \& Grobet (2001) font l'hypothèse que, bien qu'il existe des exceptions, la majorité des actes ou énoncés s'ancrent chacun sur un topique plus "pertinent » que les autres, lié par une relation d'«à propos» à l'information activée. L'analyse de la structure informationnelle consiste dès lors, dans l'approche de Roulet, Filliettaz \& Grobet (2001), à identifier le topique de chaque acte, défini comme une information déjà active (présente dans la mémoire à court terme) et comme étant l'élément «à propos » duquel l'information est activée. Ce topique peut être verbalisé linguistiquement (auquel cas la trace lexicale ou syntaxique est mise en gras), ou non (le topique est alors explicité entre parenthèses) ${ }^{2}$. Une telle analyse peut déjà être qualifiée de multidimensionnelle, dans la mesure où elle implique, outre les informations con- et cotextuelles, la prise en compte combinée de marques lexicales (formes anaphoriques ou déictiques), syntaxiques (constructions informationnellement «marquées » telles que dislocations, clivées, etc.) et prosodiques (accentuation, désaccentuation).

Une fois la structure informationnelle de chaque unité discursive analysée, il est possible de considérer le discours dans sa dimension séquentielle pour faire apparaître, selon le modèle proposé par Daneš (1974) différents types de progression informationnelle. Entre deux actes, il y aura progression à topique constant, si le topique est le même que celui qui précède, ou progression linéaire, lorsque le topique est issu de l'information qui vient d'être activée par l'unité précédente.

La progression à «thèmes dérivés » relevée par Daneš (1974), impliquant une relation d'hyperonymie entre un (hyper)thème et des sous-thèmes, n'est pas traitée à ce niveau d'analyse, car elle ne repose pas tant sur les relations de continuité s'établissant à la surface des unités discursives que sur les liens conceptuels (p. ex. partie-tout, dérivation, etc.) unissant les objets de discours. Dans le modèle de Roulet, Filliettaz \& Grobet (2001), ceux-ci peuvent être mis en évidence, dans un second temps, à travers des schématisations intitulées «structures conceptuelles » qui visent à esquisser les liens entre les différents concepts. L'approche permet enfin la mise en relation avec d'autres plans d'organisation du discours, que nous n'aborderons toutefois pas ici pour des raisons de place.

\subsection{Analyses}

\subsubsection{Analyse du discours de G. Simenon}

L'interaction dans laquelle s'inscrit le discours de G. Simenon implique un cadre topical qui peut être formulé de la manière suivante : 


\begin{tabular}{|l|l|}
\hline Locuteur 1: & Bernard Pivot, journaliste réputé, animant l'émission Apostrophes... \\
\hline Locuteur 2: & Georges Simenon, écrivain célèbre de romans et de romans policiers... \\
\hline Lieu : & Domicile de G. Simenon (il s'agit d'une émission spéciale) \\
\hline Date : & 1981 \\
\hline Evoquant : & La vie de G. Simenon, à l'occasion de la parution de ses mémoires \\
\hline Quand : & G. Simenon est en train de parler de sa manière d'écrire des romans \\
\hline
\end{tabular}

Tableau 3. Cadre topical de l'entretien entre B. Pivot et G. Simenon

Ces informations, con- et cotextuelles, constituent l'arrière-plan informationnel du discours de G. Simenon, mais elles peuvent à tout moment être mobilisées par le discours et mises à 1'avant-plan. Leur liste dans ce tableau n'est pas exhaustive, et elles sont liées à une série de représentations que l'on peut culturellement rattacher à la situation d'un écrivain, comme p. ex. " un écrivain est généralement un intellectuel », «écrire un roman demande de la réflexion », "l'écrivain a pour tâche de créer des personnages », etc. Il faut préciser que ces représentations sont certes disponibles dans les connaissances encyclopédiques, mais elles sont rendues pertinentes ici surtout parce qu'elles sont convoquées par le discours, via les présupposés liés aux négations (je ne suis pas...). La notion de cadre topical ne doit donc pas impliquer une vision statique du contexte, mais au contraire être associée à une prise en compte de la construction dynamique des informations contextuelles par le discours.

L'étape suivante consiste à analyser la structure informationnelle de chaque unité discursive, en repérant le topique explicite (ses traces sont mises en gras) ou implicite (il est restitué entre parenthèses), et à identifier la progression informationnelle impliquée.

\begin{tabular}{|l|l|l|}
\hline & Structure informationnelle & Progression \\
\hline 1 & je suis un instinctif / & \\
\hline 2 & (suis instinctif) je ne suis pas du tout un intellectuel / & Linéaire \\
\hline 3 & (instinctif) euh : je n'ai jamais pensé un roman / & Constant (instinctif) \\
\hline 4 & j'ai senti un roman \ & Linéaire \\
\hline 5 & (instinctif) je n'ai jamais pensé un personnage / & Constant (instinctif) \\
\hline 6 & j'ai senti un personnage $\backslash$ n'est-ce pas /. & Linéaire \\
\hline 7 & (instinctif) euh je n'ai jamais . inventé une situation / & Constant (instinctif) \\
\hline 8 & la situation est venue lorsque j'écrivais un roman / & Linéaire \\
\hline 9 & $\begin{array}{l}\text { (situation est venue) mais je ne savais pas du tout où mon } \\
\text { personnage allait me mener/ }\end{array}$ & Linéaire \\
\hline 10 & c'est lui qui me menait / & Linéaire \\
\hline 11 & $\begin{array}{l}\text { (me menait) et . je vivais pendant . euh d'abord onze jours /. } \\
\text { et puis à la fin sept / euh : dans la peau de ce personnage / } \\
\text { comme on dit } \backslash\end{array}$ & Constant (me menait) \\
\hline
\end{tabular}

Tableau 4. Analyse informationnelle du discours de G. Simenon 
Dans cette structure informationnelle, toutes les informations actives ou semi-actives ne sont pas prises en considération. Ainsi, le je du locuteur renvoie certes à une information rendue active par la situation, mais qui n'apparaît pas ici comme le topique le plus immédiat : dans cet extrait G, Simenon ne parle pas de luimême dans l'absolu, mais plus spécifiquement de son caractère instinctif. Par ailleurs l'analyse se base sur la prise en compte des répétitions lexicales (p. ex. la situation, ou les indéfinis un roman, un personnage qui sont ici employés dans un sens générique), de la syntaxe (voir la clivée c'est lui qui me menait) et du marquage prosodique. Sans entrer ici dans les détails du fonctionnement de l'accentuation en français, on peut noter la présence d'une insistance prosodique (détachement et accentuation) sur les verbes: pensé, senti et inventé, que l'on peut mettre en évidence à travers une analyse du son et de l'intonation par le logiciel Praat.

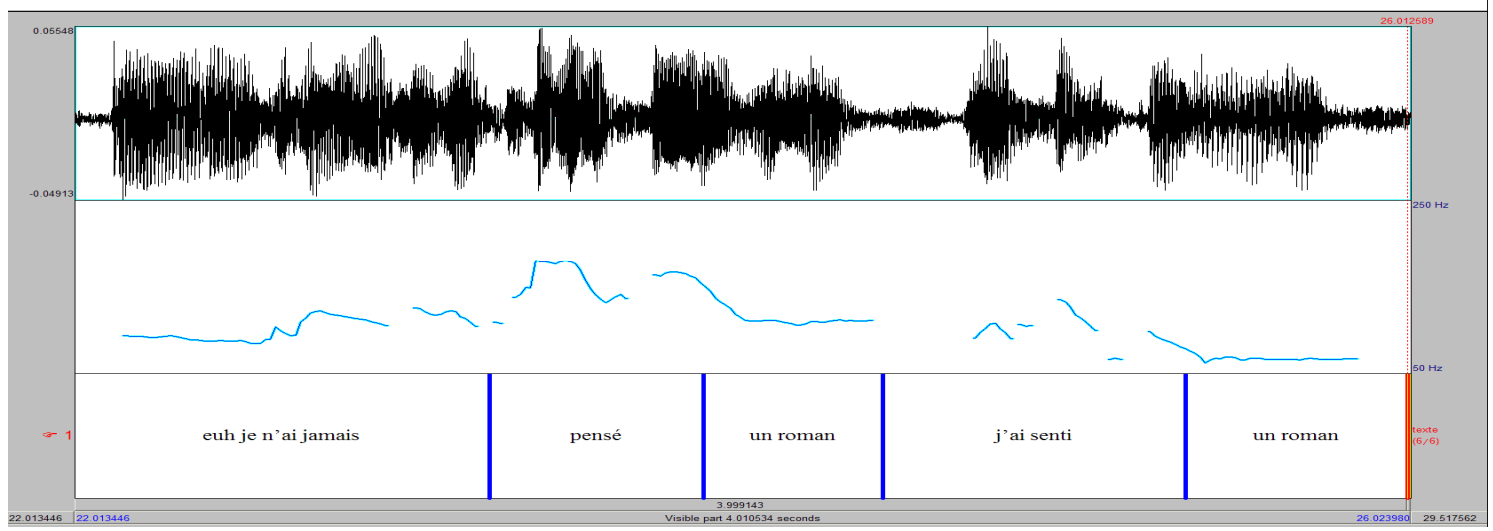

Image 1. Représentation de l'intonation à l'aide du logiciel Praat

Dans cet extrait, on observe, au niveau de l'intonation, un pic mélodique correspondant à un accent initial (Mertens 1990) sur le verbe pensé, suivi d'une chute de l'intonation ; ce même pattern est répété, mais en version atténuée et sur une hauteur plus basse, sur le verbe senti. En fait, conjointement aux parallélismes syntaxiques qui scandent la plus grande partie de ce passage, les effets de copiages et de contrastes prosodiques contribuent à souligner les oppositions qui sont ainsi mises en relief. Du point de vue de l'analyse informationnelle, ces effets d'accentuation indiquent que les verbes pensé et senti sont chaque fois réactivés.

Du point de vue des progressions informationnelles, on constate ainsi d'une part la récurrence d'enchaînements sur l'information «instinctif» (actes 2, 3, 5 et 7), et d'autre part, la présence de progressions linéaires liées à ces oppositions parallèles (1-2 je suis un instinctif / je ne suis pas du tout un intellectuel / 3-4 euh : je n'ai jamais pensé un roman / j'ai senti un roman \5-6 je n'ai jamais pensé un personnage / j'ai senti un personnage I n'est-ce pas /. 7-8 euh je n'ai jamais . inventé une situation / la situation est venue lorsque j'écrivais un roman /).

Enfin, cette analyse peut être combinée avec une représentation schématique des objets de discours, considérés du point de vue de leurs relations conceptuelles. 


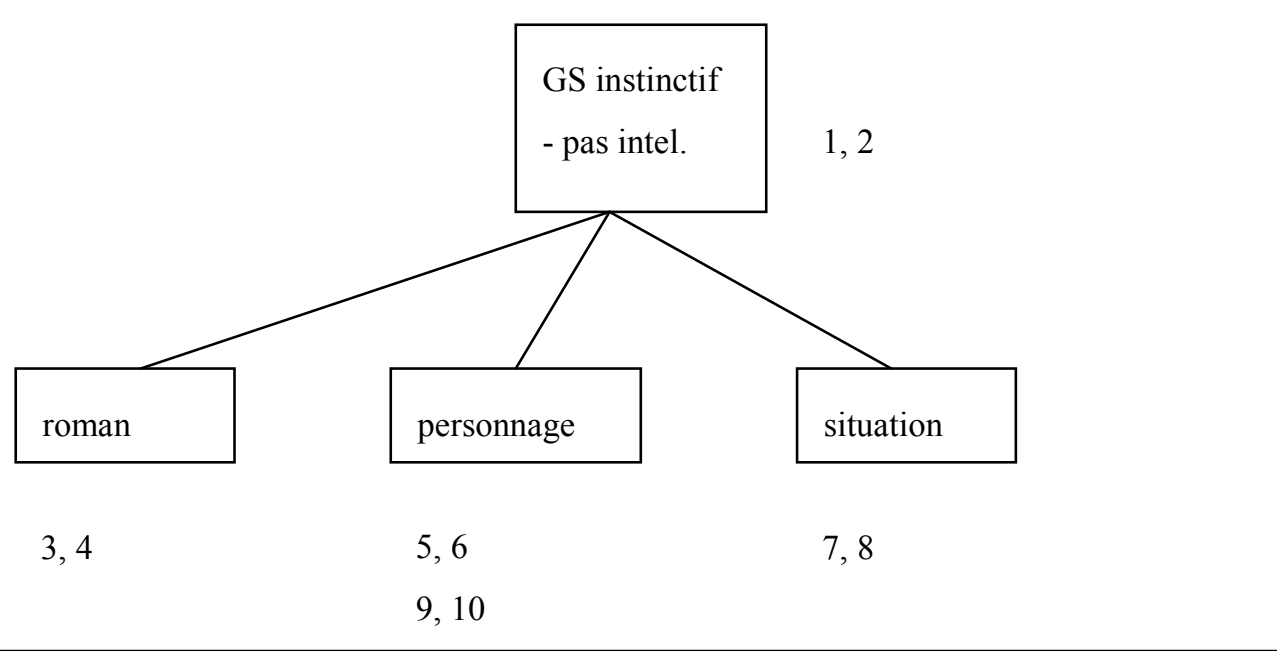

Image 2. Structure conceptuelle simplifiée du discours de G. Simenon

Cette représentation fait apparaître que cet extrait implique une progression reposant sur un objet de discours central que l'on peut reformuler comme « le caractère instinctif de l'écrivain G. Simenon ». A ce «caractère instinctif » sont associés trois exemples : la conception d'un « roman », d'un «personnage » et d'une «situation ». La prise en compte de la temporalité de la construction de ce parcours, rendue visible par la numérotation des actes, fait apparaître l'existence d'allers-retours autour de la notion de « personnage ».

En résumé, il apparaît ainsi que l'approche informationnelle conduit à mettre en relief non pas le rôle joué dans le discours par le je renvoyant au locuteur, comme le fait l'analyse positionnelle, mais plutôt les caractéristiques que ce locuteur s'attribue, ou refuse de s'attribuer.

\subsubsection{Analyse du discours de M. Foucault}

Le cadre topical du discours de M. Foucault peut être esquissé de la manière suivante :

\begin{tabular}{|l|l|}
\hline Locuteur 1: & Michel Foucault... \\
\hline Locuteur 2: & Public (étudiants) \\
\hline Lieu : & Collège de France \\
\hline Date : & 1976 \\
\hline Evoquant : & Le discours raciste et celui de la guerre des races, suite au cours précédent \\
\hline Quand : & Début du cours \\
\hline
\end{tabular}

Tableau 5. Cadre topical du discours de M. Foucault

Ce discours s'inscrit dans une interaction entre le professeur et ses étudiants, auxquels le premier attribue explicitement certaines croyances (vous avez pu croire que j'ai entrepris/la dernière fois/ de vous faire : 
l'histoire et l'éloge du discours racistell). Il faut donc à nouveau tenir compte, dans les informations d'arrière-plan, non seulement des informations liées au cadre effectif de l'interaction, mais aussi de celles qui sont activées par le discours : le locuteur construit progressivement le contexte dans lequel il ancre son discours.

Pour faciliter la comparaison avec l'analyse positionnelle, nous baserons notre analyse informationnelle sur des unités correspondantes, même si cela implique de se limiter à une analyse partielle. Cet extrait présente en effet de toute évidence un degré de complexité syntaxique supérieur au premier, ce qui peut être rattaché au contexte d'enseignement dans lequel il s'inscrit.

\begin{tabular}{|c|c|c|}
\hline & Structure informationnelle & Progression \\
\hline 1 & $\begin{array}{l}\text { bon vous avez pu croire que j'ai entrepris/ la dernière fois/ } \\
\text { de vous faire : l'histoire et l'éloge du discours racistell }\end{array}$ & \\
\hline 2 & (vous avez pu croire...) vous n'avez pas eu tout à fait tort/ & Linéaire \\
\hline 3 & $\begin{array}{l}\text { à ceci près . toutefois que ce n'est pas tout à fait du discours } \\
\text { raciste / dont j'ai voulu faire l'éloge et 1'histoire / }\end{array}$ & Linéaire (sur 1) \\
\hline 4 & $\begin{array}{l}\text { (j'ai voulu faire l'éloge et l'histoire) je dirais plutôt du } \\
\text { discours de la guerre ou de la lutte des races } \backslash\end{array}$ & Constant (éloge et histoire) \\
\hline 5 & $\begin{array}{l}\text { (plutôt du discours de la guerre ou de la lutte des races) } \\
\text { parce que je crois qu'il faut euh réserver l'expression de } \\
\text { racisme ou de discours raciste à quelque chose qui n'a été au } \\
\text { fond qu'un épisode / un épi un épisode particulier euh } \\
\text { localisé de : ce grand discours de la guerre de la lutte : des } \\
\text { races } \\
end{array}$ & Linéaire \\
\hline 6 & $\begin{array}{l}\text { le discours raciste c'en a été un épisode / une phase / c'en a } \\
\text { été à vrai dire plutôt même . le retournement / la reprise en } \\
\text { tout cas / à la fin du XIXe siècle / du discours de la guerre } \\
\text { des races / une reprise de ce vieux discours / déjà séculaire à } \\
\text { ce moment-là / dans des termes socio-biologiques / et ceci / } \\
\text { à des fins euh essentiellement de conservatisme social / et } \\
\text { dans un certain nombre de cas au moins / de domination } \\
\text { coloniale } \ \backslash\end{array}$ & Linéaire (discours raciste) \\
\hline 7 & $\begin{array}{l}\text { ceci étant dit / pour situer un peu là à la fois le lien et la } \\
\text { différence / entre discours raciste et discours de la guerre des } \\
\text { races / eh bien c'est bien l'éloge / de ce discours de la guerre } \\
\text { des races que je voulais faire / }\end{array}$ & Linéaire \\
\hline 8 & $\begin{array}{l}\text { l'éloge / en ce sens que j'aurais voulu vous montrer / euh } \\
\text { comment / pendant un temps / au moins / c'est-à-dire } \\
\text { jusqu'à la fin du XIXe siècle / jusqu'au moment où il se } \\
\text { retourne en un discours raciste / eh bien ce discours de la } \\
\text { guerre des races a fonctionné comme une contre-histoirel }\end{array}$ & Linéaire (éloge) \\
\hline 9 & $\begin{array}{l}\text { et c'est un petit peu de cette fonction de contre-histoire / } \\
\text { que je voudrais vous parler aujourd'hui }\end{array}$ & Linéaire \\
\hline
\end{tabular}

Tableau 6. Analyse informationnelle du discours de M. Foucault 
Cette analyse se fonde notamment sur le repérage d'un certain nombre d'expressions référentielles renvoyant à des informations présentées comme déjà actives ( $\mathrm{p}$. ex. la dernière fois, le discours raciste, l'éloge, cette fonction de contre-histoire), ainsi que de constructions syntaxiques marquées (p. ex. les constructions segmentées le discours raciste c'en a été..., l'éloge en ce sens...). En 5, la présence du connecteur parce que contribue également à rendre saillante l'existence d'un enchaînement linéaire. Du point de vue des progressions informationnelles, l'ensemble du passage repose ainsi sur des progressions de type linéaire, plus ou moins explicitement marquées. Toutefois, malgré la forte densité informationnelle de cet extrait, la progression de l'information est en quelque sorte ralentie par une certaine redondance au niveau des informations activées, comme c'est le cas, en 5 et en 6 , avec la répétition du fait que le discours raciste n'a été qu'un épisode du discours de la guerre des races ou, en 7 et 8 , avec la répétition de l'éloge, qui produit un effet de mise en relief.

Cet effet de piétinement, lié à de nombreuses reformulations, peut être illustré à travers une représentation schématique des objets de discours principaux, qui ne prétend toutefois pas rendre compte de la forte densité conceptuelle de cet extrait.

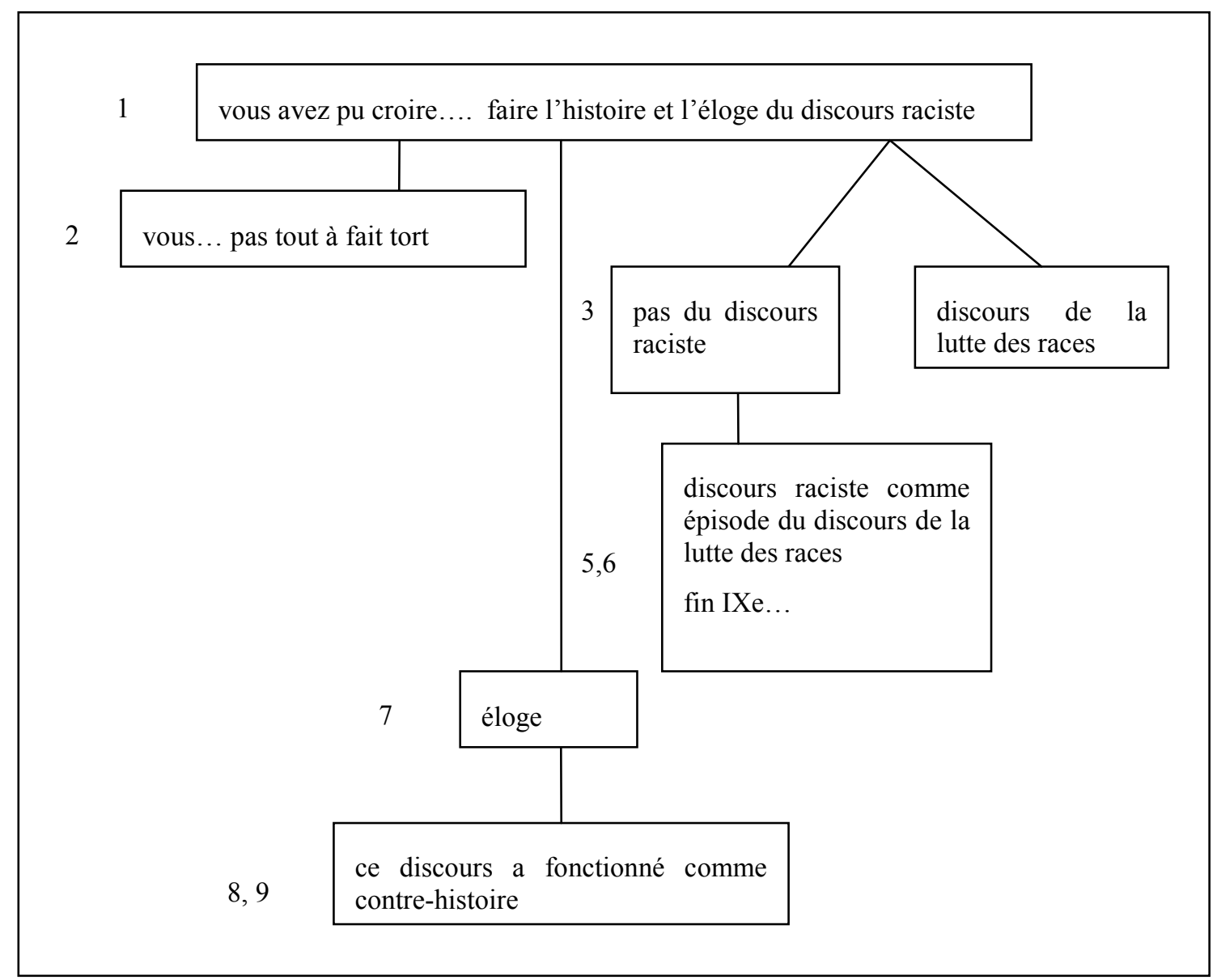

Image 3. Structure conceptuelle (très !) simplifiée du discours de M. Foucault 
Dans ce discours, un peu comme dans celui de G. Simenon, on assiste à une sorte de renversement d'informations contextuelles qui sont ici explicitement attribuées par le locuteur à son public : bon vous avez pu croire que j'ai entrepris/ la dernière fois/ de vous faire : l'histoire et l'éloge du discours racistell. Cet objet de discours, complexe, est progressivement et systématiquement déconstruit, car les différents éléments informatifs sont tour à tour commentés et reformulés : « la croyance de l'auditoire » (qui est partiellement validée en 2), « le discours raciste », qui est différencié du « discours de la lutte des races » (en 3, 4, 5 et 6), et «l'éloge », qui est justifié (en 7, 8 et 9). Comme on l'a mentionné ci-dessus, la pertinence du développement de ces objets de discours est implicitement renforcée par l'existence des stéréotypes négatifs liés au discours raciste.

\section{Conclusion}

Nous arrivons au terme de cette discussion, et pouvons maintenant reprendre les conclusions partielles données par le double éclairage positionnel et informationnel de l'organisation thématique de ces extraits de discours oraux.

Dans le fragment de G. Simenon on assiste à la négation d'une certaine image du romancier « intellectuel», négation s'inscrivant dans un dialogue ancré dans l'oral, impliquant ici une faible complexité syntaxique. Les analyses positionnelle et informationnelle donnent des résultats assez proches, puisque le Thème je est à la fois le point de départ et le « given », mais néanmoins distincts. L'éclairage positionnel fait ressortir une progression à Thème pratiquement constant sur ce je qui constitue le « support» du texte, support oral léger, pour «l'apport» qui est la notion d' « instinctif ». Du point de vue de l'approche informationnelle, le je renvoie à une information certes rendue active par le contexte, mais située à l'arrière-plan discursif. Elle se combine à d'autres informations comme celle d' " être instinctif», qui, elle, donne lieu à une progression à topique constant et permet à son tour l'introduction d'autres informations («penser un roman », "créer un personnage », etc.). L'étude des progressions fondée principalement sur la continuité informationnelle plutôt que sur la position conduit ainsi à une analyse complémentaire à la première, se situant à un niveau que l'on peut qualifier de plus spécifique dans la mesure où elle permet de décrire le développement du « caractère instinctif » de GS.

Dans le fragment de M. Foucault nous avons une reformulation progressive du « contenu », reformulation de concepts présentés dans un cours précédent, qui essaie de prendre en compte la réaction possible de l'auditoire dans un monologue formel certes oral, mais étroitement lié à l'écrit. Les éclairages positionnel et informationnel se recoupent dans la mesure où, du fait de la fonction de reformulation du fragment, le «support» (Thème comme point de départ, Halliday) et l'arrière plan informationnel (le "given », Chafe, Roulet, Filliettaz \& Grobet, etc.) sont ici les mêmes: le "vous » de l'auditoire, les propos antérieurs, ainsi que les concepts «d'éloge », d' « histoire », de « discours raciste » accompagnés de modalisations épistémiques. L'éclairage positionnel fait ainsi ressortir un support argumentatif complexe avec plusieurs Thèmes marqués dont la fonction est de repositionner les contenus du cours précédent. L'approche informationnelle conduit quant à elle à montrer comment un objet de discours initial complexe fait l'objet d'une déconstruction ainsi que d'une reformulation progressive. A la différence d'une approche purement positionnelle, l'analyse est complétée par la prise en compte des informations contextuelles et des stéréotypes mis en jeu par le discours.

Le double éclairage que nous venons de présenter nous a ainsi conduites à faire apparaître les caractéristiques formelles différentes des discours étudiés, et notamment la plus grande complexité, la densité conceptuelle et les modalisations associées au discours de M. Foucault. Néanmoins, malgré ces différences, les relations complexes et dialectiques qui s'établissent entre les dimensions positionnelle et informationelle impliquées dans l'organisation thématique se recoupent assez largement. Comme résultat préliminaire notre comparaison des deux discours oraux suggère que, pour des raisons fonctionnelles liées à la variable générique «discours public», nos deux locuteurs ont choisi consciemment ou inconsciemment ce recoupage qui donne une organisation discursive particulièrement claire et fluide.

A ce stade, il serait intéressant de poursuivre l'analyse en comparant la version transcrite du discours de M. Foucault avec le document écrit que nous mentionnons dans l'introduction. La mise en relation des 
deux documents fait en effet premièrement apparaitre la grande ressemblance entre ces deux discours, dont la plus grande partie est strictement identique, ce qui tend à confirmer l'hypothèse d'un texte lu, et deuxièmement quelques variations caractéristiques de la différence de canal: présence à l'oral d'allongements syllabiques, de marques d'hésitations ou de bribes liées à la production, ainsi que d'une réalisation prosodique distincte - les marques de production étant effacées à l'écrit et la prosodie remplacée par des signes de ponctuation. La transcription retravaillée du cours supprime également certains connecteurs tels que eh bien ou parce que, quelques répétitions lexicales et remplace certaines tournures par d'autres d'un registre plus formel (p. ex. en des termes... plutôt que dans des termes). Du point de vue de l'organisation thématique, il semble ainsi que le discours oral implique davantage de points d'appui que le discours écrit, tant au niveau positionnel qu'au niveau informationnel. Cette hypothèse préliminaire mérite toutefois d'être examinée de manière plus approfondie, en relation avec une nouvelle réflexion sur les unités discursives pertinentes, dans le cadre d'une nouvelle analyse contrastive mettant en relief les variations entre discours oral et discours écrit.

\title{
5 Annexe : extraits analysés
}

\author{
Conventions de transcription (extraits 1 et 2) \\ $/ \quad$ indication de l'intonation montante ou descendante \\ / // \II intonation continuative $(/ \backslash)$ ou conclusive $(/ / \backslash)$ \\ : $\quad$ allongements vocaliques \\ ..... pause brève, moyenne ou longue \\ Soulignements (dans l'extrait 2) passages modifiés dans la version écrite
}

1) Extrait d'un entretien télévisé entre B. Pivot et G. Simenon pour l'émission d'Apostrophes, émission spéciale sur G. Simenon enregistrée à son domicile en 1981

GS : je suis un instinctif / je ne suis pas du tout un intellectuel / euh : je n'ai jamais pensé un roman / j'ai senti un roman $\backslash$ je n'ai jamais pensé un personnage / j'ai senti un personnage $\backslash$ n'est-ce pas /. euh je n'ai jamais . inventé une situation / la situation est venue lorsque j'écrivais un roman / mais je ne savais pas du tout où mon personnage allait me mener / c'est lui qui me menait / et . je vivais pendant . euh d'abord onze jours /. et puis à la fin sept / euh : dans la peau de ce personnage / comme on dit $\backslash$

2) Michel Foucault - Notre transcription du début du cours du 2 janvier 1976 donné au Collège de France

bon vous avez pu croire que j'ai entrepris/ la dernière fois/ de vous faire : l'histoire et l'éloge du discours raciste $\backslash \backslash$ vous n'avez pas eu tout à fait tort/ à ceci près . toutefois que ce n'est pas tout à fait du discours raciste / dont j'ai voulu faire l'éloge et 1 'histoire / je dirais plutôt du discours de la guerre ou de la lutte des races $\backslash$ parce que je crois qu'il faut euh réserver l'expression de racisme ou de discours raciste à quelque chose qui n'a été au fond qu'un épisode / un épi un épisode particulier euh localisé de : ce grand discours de la guerre_de la lutte : des races $\_l$ le discours raciste c'en a été_un épisode / une phase / c'en a été à vrai dire plutôt même . le retournement / la reprise en tout cas / à la fin du XIXe siècle / du discours de la guerre des races / une reprise de ce vieux discours / déjà séculaire à ce moment-là / dans des termes socio-biologiques / et ceci / à des fins euh essentiellement de conservatisme social / et dans un certain nombre de cas au moins / de domination coloniale $\backslash$ ceci étant dit / pour situer un peu là à la fois le lien et la différence / entre discours raciste et discours de la guerre des races / eh bien c'est bien l'éloge / de ce discours de la guerre des races que je voulais faire / l'éloge / en ce sens que j'aurais voulu vous montrer / euh comment / pendant un temps / au moins / c'est-à-dire jusqu'à la fin du XIXe siècle / jusqu'au moment où il se retourne en un discours raciste / eh bien ce discours de la guerre des races a fonctionné 
comme une contre-histoire $\backslash$ et c'est un petit peu de cette fonction de contre-histoire / que je voudrais vous parler aujourd'hui.

3) Michel Foucault - Premier paragraphe du cours du 2 janvier 1976 donné au Collège de France. Transcription publiée dans Il faut défendre la société Editions Gallimard 1997: 57-58

Vous avez pu croire que j'ai entrepris, la dernière fois, de vous faire l'histoire et l'éloge du discours raciste. Vous n'avez pas eu tout à fait tort, à ceci près toutefois : ce n' est pas tout à fait du discours raciste que j' ai voulu faire l' éloge et 1'histoire, mais plutôt du discours de la guerre ou de la lutte des races. Je crois qu'il faut réserver l'expression de «racisme» ou de «discours raciste » à quelque chose qui n'a été au fond qu'un épisode, particulier et localisé, de ce grand discours de la guerre ou de la lutte des races. A vrai dire, le discours raciste n'a été qu 'un épisode, une phase, le retournement, la reprise en tout cas, à la fin du XIXe siècle, du discours de la guerre des races, une reprise de ce vieux discours, déjà séculaire à ce moment-là, en des termes socio-biologiques, à des fins essentiellement de conservatisme social et, dans un certain nombre de cas au moins, de domination coloniale. Cela étant dit pour situer, à la fois, le lien et la différence entre discours raciste et discours de la guerre des races, c'est bien l'éloge de ce discours de la guerre des races que je voulais faire. L'éloge, en ce sens que j'aurais voulu vous montrer comment, pendant un temps au moins - c'est-à-dire jusqu'à la fin du XIXe siècle, jusqu'au moment ou il se retourne en un discours raciste -, ce discours de la guerre des races a fonctionné comme une contrehistoire. Et c'est un petit peu de cette fonction de contre-histoire que je voudrais vous parler aujourd'hui.

\section{Références bibliographiques}

Berry M. (1996). What is Theme? A(nother) Personal View. In Berry M., Butler C., Fawcett R., et Huang G. (Eds) Meaning and Form: Systemic Functional Interpretations - Meaning and Choice in Language: Studies for Michael Halliday. Stamford : Ablex Publishing Corporation, 5-64.

Brown, G. \& Yule, G. (1983). Discourse Analysis. Cambridge : Cambridge University Press.

Chafe, W. (1994). Discourse, Consciousness, and Time. Chicago et Londres : The University of Chicago Press.

Davies F. (1997). Marked Theme as a heuristic for analysing text-type, text and genre. In Pique J. et Viera D.J. (Eds.) Applied Linguistics : Theory and Practice in ESP. Valencia : Valencia University Press, 45-79.

Daneš F. (1974) Functional sentence perspective and the organization of the text. In F. Daneš (ed.) Papers on Functional Sentence Perspective Czech Academy of Science, Prague : Mouton Academic Publishing House, 106128.

Firbas, J. (1964). On defining the Theme in Functional Sentence Analysis. Travaux Linguistiques de Prague 1, 267280.

Firbas J. (1992). Functional sentence perspective in written and spoken communication. Cambridge : Cambridge University Press.

Fries P. (1983). On the status of Theme in English : arguments for discourse. In Petöfi J.S. et Sozer E. (Eds) Micro and Macro Connexity of Texts : Papers in Text Linguistics. Hambourg : Helmut Buske, 116-152.

Gadet, F. (1996). Une distinction bien fragile : oral/écrit. Tranel 25, 13-25.

Galmiche, M. (1992). Au carrefour des malentendus : le thème. L'information grammaticale 54, 3-10.

Halliday, M.A.K. (1967). Notes on transitivity and theme in English. Journal of Linguistics 3.1, 37-81, 3.2, 199-244.

Halliday M.A.K. (1994). An Introduction to Functional Grammar. Londres : Edward Arnold Publisher.

Halliday M.A.K. et Matthiessen C.M.I.M (2004). An Introduction to Functional Grammar. Londres : Edward Arnold Publisher. 
Lambrecht, K. (1994). Information Structure and Sentence Form. Topic, Focus and the Mental Representation of Discourse Referent. Cambridge : Cambridge University Press.

Mendenhall, V. (1990). Une introduction à l'analyse du discours argumentatif. Ottawa : Les Presses de l'Université.

Mertens, P. (1990). Intonation. In Blanche-Benveniste, C. et al. (1990) Le français parlé. Etudes grammaticales. Paris : CNRS, 159-176.

Mondada, L. (1994). Verbalisation de l'espace et fabrication du savoir. Approche linguistique de la construction des objets de discours. Lausanne : Université de Lausanne.

Montemayor Borsinger, A. (2009). Tema : Una perspectiva funcional de la organización del discurso. Buenos Aires : Editorial Universitaria de Buenos Aires.

Roulet, E., Filliettaz, L. \& Grobet, A. (2001). Un modèle et un instrument d'analyse de l'organisation du discours, Berne : Lang.

Stainton C. (1993). Metadiscourse and the Analytical Text: a Genre-Based Approach to Children's Written Discourse. Thèse de doctorat, Manchester : Université de Manchester.

Weil, H. (1844) De l'ordre des mots dans les langues anciennes comparées aux langues modernes. Paris : Librairie A. Franck.

${ }^{1}$ Pour distinguer les approches, nous utiliserons dorénavant le terme de «topique » pour marquer un point de vue informationnel, par opposition au « Thème » utilisé dans la perspective positionnelle.

${ }^{2}$ Ces conventions, proposées dans Roulet, Filliettaz \& Grobet (2001), sont mises en oeuvre dans les tableaux 4 et 6. 